\title{
Strange and non-strange distributions from the collider data
}

\author{
Sergey Alekhin*† \\ II. Institut für Theoretische Physik, Universität Hamburg, Luruper Chaussee 149, D-22761 \\ Hamburg, Germany; \\ Institute for High Energy Physics, 142281 Protvino, Russia \\ E-mail: sergey.alekhin@desy.de \\ Johannes Blümlein \\ Deutsches Elektronensynchrotron DESY, Platanenallee 6, D-15738 Zeuthen, Germany \\ E-mail: Johannes.Bluemlein@desy.de
}

\section{Sergey Kulagin}

Institute for Nuclear Research of the Russian Academy of Sciences, Moscow 117312, Russia

E-mail: kulagin@ms2.inr.ac.ru

\section{Sven-Olaf Moch}

II. Institut für Theoretische Physik, Universität Hamburg, Luruper Chaussee 149, D-22761

Hamburg, Germany

E-mail: sven-olaf.moch@desy.de

\section{Roberto Petti}

Department of Physics and Astronomy, University of South Carolina, Columbia SC 29208, USA

E-mail: roberto.petti@cern.ch

We check the stability of the ABMP16 fit with respect to modifications of quark PDFs suggested in the recent literature: the strange sea enhancement and a positive non-vanishing $d / u$ ratio at $x \rightarrow 1$. These possibilities are examined using test versions of the ABMP16 PDF fit which demonstrate no need of those changes. Furthermore, we localize peculiar features in other analyses which are responsible for a different behaviour of the PDFs obtained. The strange sea enhancement can be explained by a choice of the PDF shapes being not flexible enough and therefore leading to an over-suppressed $d$-quark distribution. This suppression has to be compensated by a corresponding rise by the $s$-quark distribution. As a result, an unusually large strange sea suppression factor is obtained. The non-vanishing value of $d /\left.u\right|_{x=1}$ becomes consistent with zero in case the higher-order QCD corrections to the $\mathrm{D} \varnothing W$-asymmetry data, which drive its value, are taken into account. Furthermore, the related $e$-asymmetry sample prefers a slightly negative value of $d /\left.u\right|_{x=1}$, although it is consistent with zero. These clarifications support confidence in the PDF shapes used in the ABMP16 analyses.

XXVI International Workshop on Deep-Inelastic Scattering and Related Subjects (DIS2018)

16-20 April 2018

Kobe, Japan

\footnotetext{
* Speaker.

${ }^{\dagger}$ This work was supported in part by Bundesministerium für Bildung und Forschung (contract 05H15GUCC1) andx by the Russian Science Foundation grant No. 14-22-00161.
} 
The quark distributions in the nucleon are in general well constrained due to the experimental information from deep-inelastic-scattering (DIS) of leptons off nucleons. Here the most important data are a combination of inclusive neutral-current (NC) scattering data off proton and deuteron targets and data on semi-inclusive charged-current (CC) $c$-quark production. However, such an approach does not work at small Bjorken $x$, where only electron-proton inclusive data, accumulated at HERA, are available. On the other hand, the fast emerging samples of the Drell-Yan (DY) data, collected at the LHC, provide additional constraints on the parton distribution functions (PDFs), particularly on the quark ones. These samples, together with the DY data obtained at the Tevatron collider in the forward region, probe PDFs at values of $x$ down to $10^{-4}$ and therefore provide a supplementary constraint to the HERA inclusive data. This potential was in particular employed in the ABMP PDF fit in order to disentangle small- $x u$ - and $d$-quark distributions [1,2]. The impact of the collider DY data on this piece is very significant. Indeed, in the variant of ABMP16 fit, which does not include this data sample, the small- $x$ quark distributions are quite uncertain and the quark iso-spin asymmetry $(\bar{d}-\bar{u}) /(\bar{d}+\bar{u})$ is in broad agreement with 0 at small $x$, while in the nominal ABMP16 fit it is clearly negative at $x \sim 10^{-4}$, see Fig. 1 . The strange sea determination is also improved due to collider DY data, however, in this case the effect is less pronounced since the strange sea is constrained to a great extent by the neutrino-induced DIS data on charm production by the $\mathrm{CCFR} / \mathrm{NuTeV}$ and NOMAD experiments $[3,4]$, which are particularly sensitive to the strange sea contribution.
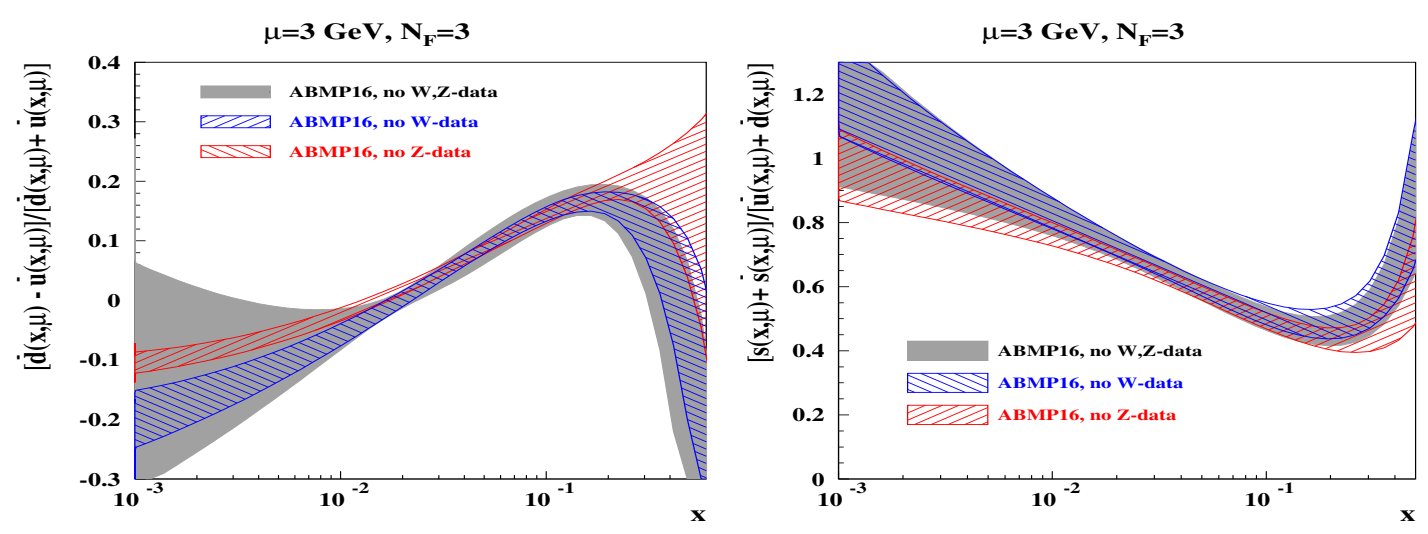

Figure 1: The $1 \sigma$ band for the NNLO quark iso-spin asymmetry $(\bar{d}-\bar{u}) /(\bar{d}+\bar{u})$ (left) and the strange sea suppression factor $(s+\bar{s}) /(\bar{d}+\bar{u})$ (right) in the 3 -flavor scheme at the scale of $\mu=3 \mathrm{GeV}$ as a function of Bjorken $x$ obtained in the variants of the ABMP16 PDF fit [2] with the data on production of $W$-bosons (left-tilted hash), $Z$-bosons (right-tilted hash), and both $W$ - and $Z$-bosons (shaded area) excluded form the fit.

This is in contrast to the PDF fit of Ref. [5] based on the combination of the recent highstatistics ATLAS data on $W$ - and $Z$-boson production with the HERA data on inclusive DIS. In the ATLAS analysis the strange sea is determined by a combination of various constraints coming from the inclusive data and as a result it is found to be substantially enhanced as compared to the one obtained in the ABMP16 fit. This difference might be in principle explained by impact of the ATLAS data [5], which are not included into the ABMP16 fit, and the different PDF shapes used in these two analyses. The ATLAS fit essentially follows the earlier HERAPDF framework derived for 
the analysis of the HERA data alone and, therefore, including many constraints on PDFs, which allow a sensible PDF determination using a limited set of the inclusive DIS data. To check the impact of such a choice of the PDF shape we perform a variant of the ABMP16 fit [7] with the epWZ16 PDF shape, which is employed in the ATLAS analysis. Furthermore, in order to separate the influence of the experimental input we discard all DY collider data, including the earlier data from ATLAS [6], which are used in the ABMP16 analysis, however. At the same time we add to the analysis data on DIS off deuteron targets allowing to disentangle the quark distributions after discarding the collider DY data. We account for the deuteron wave-function effects, relevant in this case, by applying the Kulagin-Petti model [8], which is based on the microscopic description in terms of the off-shell nucleon function. Such an approach provides universal description of the nuclear effects in a wide range of targets and the PDFs extracted within this framework are consistent with the ones preferred by the existing DY collider data [9]. It turns out that, despite the ATLAS data are not used, the strange sea is still enhanced as compared to the nominal ABMP16 PDFs, see Fig. 2. It is worth noting that the charm production data [3, 4] are well described in this variant of fit, with $\chi^{2} / N D P=167 / 226$ achieved $^{1}$. It is interesting to see that the strange sea
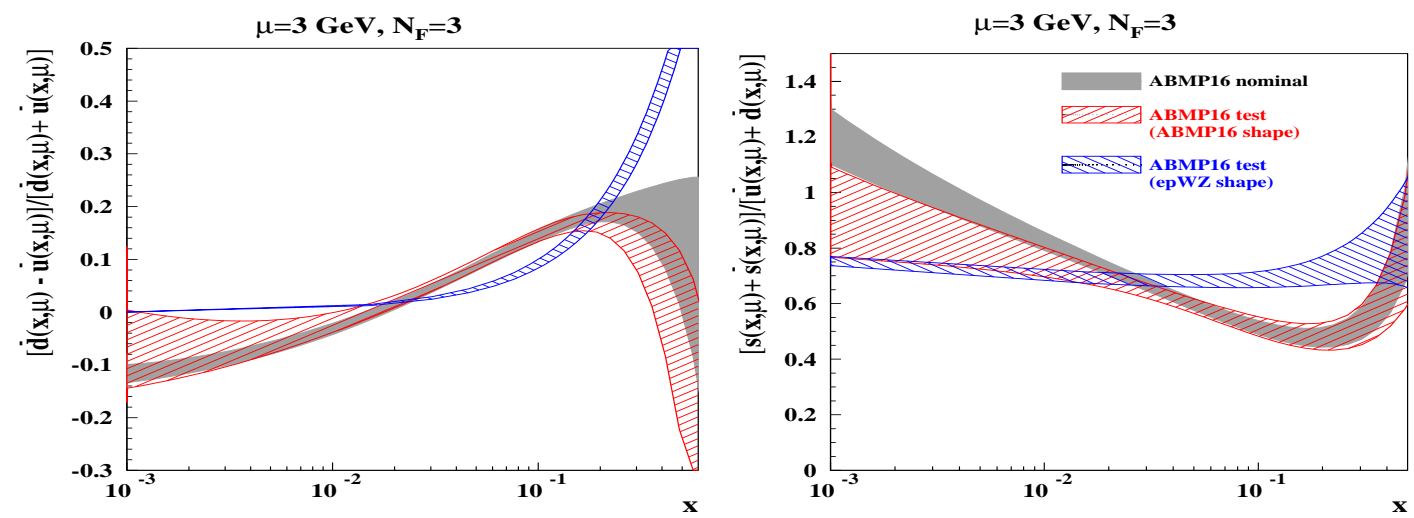

Figure 2: The same as in Fig.1 for the test variants of the ABMP16 fit with the collider DY data excluded, the DIS deuteron data added and various PDF shapes used (left-tilted hash: epWZ16 shape, right-tilted hash: nominal ABMP16 shape) in comparison with the nominal ABMP16 PDFs (shaded area).

distribution obtained with the ABMP16 PDF shape and the same data selection is significantly smaller at $x \sim 0.1$ and agrees with the nominal ABMP16 PDFs. However, the value of $\chi^{2} / N D P=$ $161 / 226$ obtained in this case for the CCFR/NuTeV and NOMAD data is very similar to the one for the fit with epWZ16 shape. Such a peculiarity is explained by the fact that the epWZ16 variant of the fit suggests simultaneous suppression of the $d$-quark distribution at $x \sim 0.1$, see Fig. 2 . This happens due to the evidently over-constrained fit in the epWZ16 shape of the quark iso-spin asymmetry, which by construction vanishes at $x \rightarrow 0$ and follows this trend at larger $x$. However, this is in disagreement with the FNAL-E-866 experiment on the muon pair production in protonproton and proton-deuteron scattering [10], which clearly reports a positive value of $(\bar{d}-\bar{u})$ at this kinematics. As a result, the description of the E-866 data obtained in the test fit with the epWZ16

\footnotetext{
${ }^{1}$ The value of $\chi^{2}$ for the CCFR/NuTeV data is smaller than the number of data points (NDP) due to the definition of the experimental covariance matrix, which includes effects of neighbor bin correlations, so that the effective number of degrees of freedom is smaller than the number of data points, see Ref. [3] for details.
} 
shape is quite poor giving the value of $\chi^{2} / N D P=96 / 39$ to be compared to $49 / 39$ obtained in the variant of fit with ABMP16 PDF shape. This brings us to the conclusion that the epWZ16 PDF shape is not flexible enough to study the strange sea and tends to pull it up at the price of a suppressed $d$-quark distribution.

For a further check of the impact of the ATLAS data on the strange sea we perform a variant of fit based on a combination of the ATLAS and inclusive HERA data with the ATLAS data on $W$ - and $Z$-boson production $^{2}$. Such a selection of data is in line with the ATLAS analysis of Ref. [5]. However, in this case we use a more flexible ABMP16 PDF shape. The strange sea obtained in this way is in a broad agreement with the ATLAS result due to quite large uncertainties at $x \gtrsim 0.1$, see Fig. 3. This means the combination of the ATLAS and HERA data is not sufficient to disentangle the distributions for all quark species. Meanwhile, one can improve the fit by adding the E-866 data serving as an additional constraint on the quark iso-spin asymmetry $(\bar{d}-\bar{u})$. Due to this input the strange sea uncertainty reduces substantially, while the central value is still in agreement with the ABMP16 result. The latter is

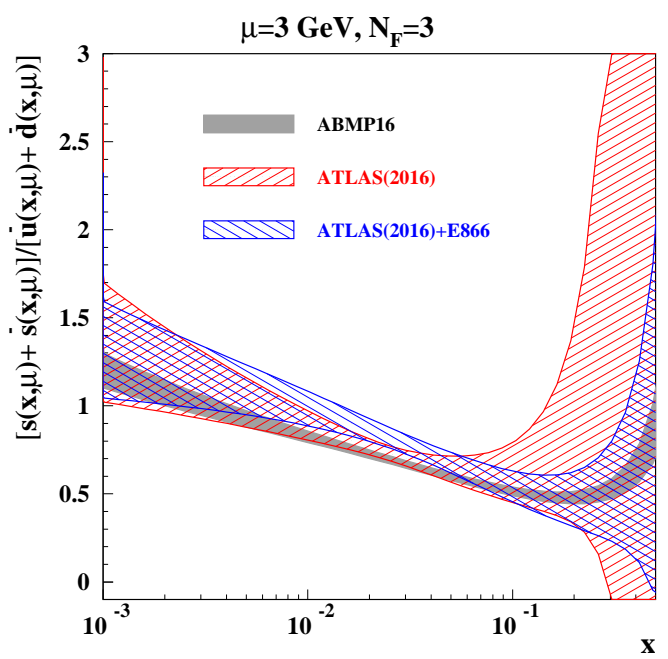

Figure 3: The same as in Fig.1 for the strange sea suppression factor $(s+\bar{s}) /(\bar{d}+\bar{u})$ obtained in the test variants of the ABMP16 fit with ATLAS data used in combination with the inclusive HERA data (lefttilted hash) and the E-866 data on the top (right-tilted hash) in comparison with the nominal ABMP16 PDFs (shaded area). driven to a great extent by the CCFR/NuTeV and NOMAD data. Here no tension between E-866 and ATLAS data is observed, obtaining the values of $\chi^{2} / N D P=48 / 39$ and $40 / 34$, respectively ${ }^{3}$. The strange sea distribution obtained in this way is in reasonable agreement with the one obtained in the ABMP16 fit. The small enhancement still observed at $x \sim 0.01$ is driven by the $Z$-boson data, which deserve further study in view of some tension with the CMS results, see Ref. [7] for details.

Another debatable issue concerns the ratio $d / u$. Due to the lack of experimental data it is poorly constrained at $x \sim 1$. Therefore some uncertainty in $\lim _{x \rightarrow 1} d / u$ persists. In particular, as it was found in a recent NLO analysis by CJ15 [11] $d / u$ is finite at $x \rightarrow 1$, in contrast to the asymptotic value $d / u \rightarrow 0$ commonly suggested by various PDF sets available. The CJ15 fit is based on a combination of various DIS, DY, and jet production data with the crucial role for the determination of $d / u$ at large $x$ played by the DØ results on the $W$-boson charge asymmetry [12]. The NLO QCD corrections to this process are computed in Ref. [11] using the $K$-factor approach. However, since for the $\bar{p} p$ initial state of the DØ experiment the cross sections of $W^{+}$

\footnotetext{
${ }^{2}$ We take only the high-statistics $Z$-boson data of the central-rapidity region into account and leave out the less accurate data in the forward rapidity region.

${ }^{3}$ The ATLAS value of $\chi^{2}$ is better than the one obtained for the respective sample of the ATLAS data in the analysis of Ref. [5].
} 
and $W^{-}$coincide, the respective $K$-factors cancel in the charge asymmetry and the NLO correction factors appear equal to one, i.e. just the LO approximation is reproduced in such a manner.

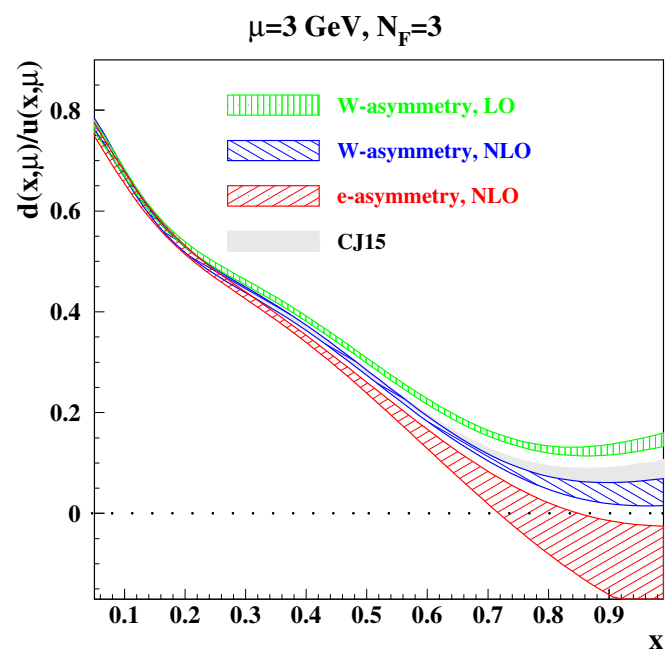

Figure 4: The same as in Fig.2 for the ratio $d / u$ obtained using the CJ15 PDF shape [11] and with addition of the $\mathrm{D} \varnothing$ data on $W$ - and $e$-asymmetry, described within various approximations (vertical hash: $W$-asymmetry [12] at LO, left-tilted hash: the same in the NLO, right-tilted hash: $e$-asymmetry [13] at NLO) in comparison with the nominal CJ15 PDFs (shaded area).

behavior since the data sets used are not sensitive to this region.

The ratio $d / u$ obtained in the variant of this test fit with the LO approximation employed for the description of the $W$-asymmetry data demonstrates a rising trend at large $x$ resulting into $\left.(d / u)\right|_{x=1}=0.15 \pm 0.01$, in line with the CJ15 finding, see Fig. 4. However, with account of the NLO corrections it takes essentially lower values with $\left.(d / u)\right|_{x=1}=0.04 \pm 0.03$. This is almost consistent with zero within $1 \sigma$. Note that the uncertainties in $d / u$ rise essentially from LO to NLO. This is obviously related to the involvement of gluon-initiated processes appearing in the latter case and the impact of the gluon distribution uncertainty propagating through this channel. One more aspect of this study is a selection between the $\mathrm{D} \emptyset$ data on the $W$-asymmetry and the $e$-asymmetry [13]. Both sets are derived from the same sample of events and the latter is, in fact, directly measured in experiment, while the $W$-asymmetry is obtained by processing this primary information. Such a processing requires a certain modeling and the result of this is sensitive to the PDFs used. Moreover, the PDFs obtained from the analysis of the $e$-asymmetry data are not consistent with the data on the $W$-asymmetry, see Fig. 9 in Ref. [1]. Motivated by this bias we also perform a variant of test fit with the $e$-asymmetry data instead of the $W$-asymmetry. The NLO corrections are applied in order to provide the relevant theoretical accuracy. The $d / u$ ratio obtained in this way goes further down with even larger uncertainties, see Fig. 4, and the value $\left.(d / u)\right|_{x=1}=-0.12 \pm 0.09$ is consistent with zero within the uncertainties. Comparing it with the 
value obtained from the $W$-asymmetry we find a broad agreement, however, the $e$-asymmetry result is less sensitive to model assumptions, as we mentioned before. In any case, the CJ15 result on $d / u$ at large $x$ is obviously due to a particular selection of the data and the limited theoretical accuracy of the analysis. Otherwise we arrive at the value of $\left.(d / u)\right|_{x=1}$ consistent with zero which justifies such a constraint commonly imposed in the PDF fits.

In conclusion, we considered modifications of quark PDFs suggested in the recent literature: the strange sea enhancement [5] and a positive non-vanishing $d / u$ ratio at $x \rightarrow 1$ [11]. These possibilities were examined using test versions of the ABMP16 PDF fit [2], which demonstrate no need of those changes. Furthermore, we localize peculiar features of these analyses which are responsible for the uncommon behavior of their PDFs obtained. The strange sea enhancement [5] can be explained by non-flexible PDF shapes leading to an over-suppressed $d$-quark distribution. This suppression has to be compensated by the respective rise of the $s$-quark distribution. As a result an unusually large strange sea suppression factor is obtained. The non-vanishing value of $d /\left.u\right|_{x=1}$ [11] becomes consistent with zero in case the NLO corrections to the $\mathrm{D} \emptyset W$-asymmetry data are taken into account. Furthermore, the related $e$-asymmetry sample prefers a slightly negative value of $d /\left.u\right|_{x=1}$, although being consistent with zero. These clarifications support confidence in the PDF shape used in the ABMP16 fit.

\section{References}

[1] S. Alekhin, J. Blümlein, S. Moch and R. Plačakytè, Phys. Rev. D 94 (2016) 114038 [arXiv:1508.07923 [hep-ph]].

[2] S. Alekhin, J. Blümlein, S. Moch and R. Plačakytè, Phys. Rev. D 96 (2017) 014011 [arXiv:1701.05838 [hep-ph]].

[3] M. Goncharov et al. [NuTeV Collaboration], Phys. Rev. D 64 (2001) 112006 [hep-ex/0102049].

[4] O. Samoylov et al. [NOMAD Collaboration], Nucl. Phys. B 876 (2013) 339 [arXiv:1308.4750 [hep-ex]].

[5] M. Aaboud et al. [ATLAS Collaboration], Eur. Phys. J. C 77 (2017) 367 [arXiv:1612.03016 [hep-ex]].

[6] G. Aad et al. [ATLAS Collaboration], Phys. Rev. D 85 (2012) 072004 [arXiv:1109.5141 [hep-ex]].

[7] S. Alekhin, J. Blümlein and S. Moch, Phys. Lett. B 777 (2018) 134 [arXiv:1708.01067 [hep-ph]].

[8] S. A. Kulagin and R. Petti, Nucl. Phys. A 765 (2006) 126 [hep-ph/0412425].

[9] S. I. Alekhin, S. A. Kulagin and R. Petti, Phys. Rev. D 96 (2017) 054005 [arXiv:1704.00204 [nucl-th]].

[10] R. S. Towell et al. [NuSea Collaboration], Phys. Rev. D 64 (2001) 052002 [hep-ex/0103030].

[11] A. Accardi, L. T. Brady, W. Melnitchouk, J. F. Owens and N. Sato, Phys. Rev. D 93 (2016) 114017 [arXiv:1602.03154 [hep-ph]].

[12] V. M. Abazov et al. [D0 Collaboration], Phys. Rev. Lett. 112 (2014) no.15, 151803 Erratum: [Phys. Rev. Lett. 114 (2015) 049901] [arXiv:1312.2895 [hep-ex]].

[13] V. M. Abazov et al. [D0 Collaboration], Phys. Rev. D 91 (2015) no.3, 032007 Erratum: [Phys. Rev. D 91 (2015) 079901] [arXiv:1412.2862 [hep-ex]].

[14] S. Alekhin, J. Blümlein and S. Moch, Eur. Phys. J. C 78 (2018) 477 [arXiv:1803.07537 [hep-ph]]. 